\title{
Partially Collapsed Parallel Gibbs Sampler for Dirichlet Process Mixture Models
}

\author{
Halid Ziya Yerebakan**, Murat Dundar \\ Computer and Information Science \\ IUPUI \\ Indianapolis, IN 46202
}

\section{ABSTRACT}

\begin{abstract}
Dirichlet Process (DP) is commonly used as a non-parametric prior on mixture models. It has adaptive model selection capability which is useful in clustering applications. Although exact inference is not tractable for this prior, Markov Chain Monte Carlo (MCMC) samplers have been used to approximate the target posterior distribution. These samplers often do not scale well. Thus, recent studies focused on improving run-time efficiency through parallelization. In this paper, we introduce a new sampling method for DP by combining Chinese Restaurant Process (CRP) with stick-breaking construction allowing for parallelization through conditional independence at the data point level. Stick breaking part uses an uncollapsed sampler providing a high level of parallelization while the CRP part uses collapsed sampler allowing more accurate clustering. We show that this partially collapsed Gibbs sampler has significant advantages over the collapsed-only version in terms of scalability. We also provide results on real-world data sets that favorably compares the proposed inference algorithm against a recently introduced parallel Dirichlet Process samplers in terms of F1 scores while maintaining a comparable run-time performance.
\end{abstract}

(c) 2017 Elsevier Ltd. All rights reserved.

\section{Introduction}

The Bayesian way to address model selection problem in finite mixture models is to have a prior distribution over the models. Dirichlet Process (Ferguson 1973) prior is a powerful choice that serves for this purpose by inferring the number of mixture components from the data. Thanks to its consistency property a DP prior can estimate the true density of the data as the number of data points grows to infinity.

Model learning in DP mixture models often achieved either by variational Bayes methods or MCMC sampling algorithms. Variational Bayes methods mostly utilize stick-breaking representation (Ishwaran and James, 2001) for inference. In this representation, the number of sticks is truncated to obtain a tractable algorithm. On the other hand, MCMC-based methods mostly utilize Chinese Restaurant Process (CRP) representation that collapses out cluster parameters to reduce the number of parameters to be sampled. CRP creates dependencies among data points, which requires inference algorithms that operate sequentially. Thus, CRP representation often does not scale well to big datasets. In this paper, we utilized a useful representation of Dirichlet Process that couples stick-breaking and

\footnotetext{
${ }^{* *}$ Corresponding author

e-mail: hzyereba@umail.iu.edu ( Halid Ziya Yerebakan)
}

CRP representations together. Stick-breaking part leads to an uncollapsed sampler that enables parallelization at the data point level. CRP part leads to a collapsed sampler that eliminates the need for fixing the number of sticks in advance. We propose a partially-collapsed Gibbs sampler that combines these two into a parallelizable inference algorithm. We compared our algorithm with standard collapsed Gibbs sampler, parallel split-merge sampler (Chang and Fisher III, 2013) and parallel slice sampler Ge et al. (2015).

Our contributions can be summarized as follows. Unlike the decomposition approaches in the literature (Williamson et al. 2013; Lovell et al., 2012) that use identical distributions, our approach produces non-identical distributions, which is essential for more balanced distribution of the computational load across processing cores. We introduce an inference algorithm that combines stick-breaking based uncollapsed Gibbs sampler with a CRP-based collapsed sampler. Uncollapsed sampler prompts more significant moves in the state space while enabling parallel sampling by establishing conditional independence between data points. Collapsed sampler generates new clusters by being more adaptive to small clusters. We performed experiments on realworld datasets evaluating clustering accuracy and efficiency of the proposed inference algorithm in terms of $F_{1}$ score and run time, respectively. We observed up to an order of magnitude 
speed up on an eight-core machine compared to a collapsedonly sampler. We also compare our results against a recently introduced parallel samplers and report higher $F_{1}$ scores and favorable run-time performance.

In the rest of the paper, we first discuss related work on MCMC samplers for DP mixture models. Next, we review Dirichlet Process and relevant representations. Then, we introduce the hybrid representation for DP and propose a parallel Gibbs sampler. Finally, we present our experimental results and conclude by summarizing our contributions and offering insight for future research.

\section{Related Work}

We review related works in the area of MCMC inference algorithms for DP mixture models as this line of work is the most relevant to the proposed inference algorithm. Different variants of Gibbs sampler can be used for inference with DP mixture models (Neal, 2000, Ishwaran and James, 2011). If the data model is conjugate to the base distribution, cluster parameters can be integrated out, which collapses the inference directly onto the cluster indicator variables. This version of Gibbs sampler is known as the collapsed Gibbs sampler in the literature. Collapsed Gibbs sampler is inherently sequential as integrating out cluster parameters creates dependencies between data points. The convergence with the collapsed Gibbs sampler is slow in general as the sampler makes slow incremental moves in each scan.

Alternative sampling schemes based on Metropolis-Hastings are available for non-conjugate models (Neal, 2000, Jain et al., 2007; Görür and Rasmussen, 2010). Jain et al. (Jain and Neal, 2004) propose a split-merge algorithm to improve convergence and alleviate the local optima problem. Dahl (Dahl, 2003) reduces computational burden using conjugate models. However, each split-merge proposal still requires a high volume of computation. Furthermore, when the move is rejected the calculations are completely lost.

The common characteristic of all these inference techniques is that they require sequential processing, which does not readily allow for parallelization and thus does not scale well to big datasets. Parallelization using modern hardware is a promising opportunity to obtain more practical algorithms. The naive approach to parallelizing Dirichlet Process inference would involve running multiple independent samplers. More samples could be obtained this way than a single sampler in the same amount of time producing more accurate predictions. However, as all samplers would require a burn-in time the computational gain by running several independent samplers would be limited.

More recent studies focus on parallelizing a single sampler to speed up inference (Lovell et al. 2012, Williamson et al. 2013). These methods decompose a single Dirichlet Process into several identical DPs and derive exact samplers. However, using identical DPs makes these algorithms vulnerable to load balancing issues (Gal and Ghahramani, 2013). Chang et. al. (Chang and Fisher III 2013) propose an algorithm that allows parallelization at the data point level. Conditioned on the cluster parameters data points become independent of each other and likelihood computations can be carried out in parallel. To ensure ergodicity the algorithm uses split-merge moves (Jain and Neal, 2004). Split-merge proposals are efficiently computed by introducing auxiliary variables. This approach does not suffer much from load balancing issues as likelihood computations for all data points can be equally distributed among processing cores.

However, split-merge calculations are not the only way to ensure ergodicity. Besides, split-merge evaluation can be very costly. Chang et. al. (Chang and Fisher III, 2013) evaluate one random split proposal on the average in every hundred scans in their implementation. Although this speeds up inference, the effect of split-merge steps in the overall inference could be limited. We use a simpler and intuitive way to achieve ergodicity by preserving the uncollapsed part in the sampler while replacing split-merge moves with a collapsed sampler. Our proposed approach is also related to the retrospective sampler introduced in (Papaspiliopoulos and Roberts, 2008) with the main difference being the collapsed sampler we use to handle new clusters as opposed to retrospective generation of new clusters using stickbreaking by retrospective sampler. Additionally, retrospective sampler is not tested for parallel inference, which happens to be the main focus of our study.

Another related work uses a similar decomposition of DP as ours during inference (Ge et al., 2015). This work utilizes slice sampling to avoid representing infinite dimensional discrete distributions. This is achieved by introducing slice variables into the uncollapsed sampler. This approach can potentially enable very high level of parallelization. However, experimental results suggest sampler mixes poorly in high dimensions due to low probability of the points to chose proposed new clusters.

\section{Background}

We start by introducing Dirichlet Process and its relevant representations. The formal definition uses marginalization of Dirichlet Process into a finite number of partitions as given in equation (1).

Definition 1 (Dirichlet process). For a measurable space $\Omega$, a base probability distribution $H$, and a positive concentration parameter $\alpha$, the Dirichlet process $D P(\alpha H)$ is a stochastic process that generates a measure $G$ following the marginal property for any finite partition $\left(A_{1}, \ldots, A_{K}\right)$ of $\Omega$

$$
\left(G\left(A_{1}\right), \ldots, G\left(A_{K}\right)\right) \sim \operatorname{Dir}\left(\alpha H\left(A_{1}\right), \ldots, \alpha H\left(A_{K}\right)\right),
$$

We denote the generative model by $G \sim D P(\alpha H)$. Although $G$ consists of a countably infinite number of discrete probability masses called atoms, finite data requires only finite number of atoms. The effective number of atoms used in the representation could grow with the size of the data creating a non-parametric structure. This structure provides a distribution over mixture models with unknown number of components.

\subsection{Stick-Breaking Process}

A sample from the Dirichlet Process could be created using the finite-dimensional marginal property of DP. Sethuraman proved a constructive definition of DP (Sethuraman, 1991) known as the stick-breaking representation (2). 


$$
\begin{aligned}
\beta_{j}^{\prime} & \sim \operatorname{Beta}(1, \alpha) \\
\theta_{j} & \sim H \\
w_{j} & =\beta_{j}^{\prime} \prod_{l=1}^{j-1}\left(1-\beta_{l}^{\prime}\right) \\
G & =\sum_{j=1}^{\infty} w_{j} \delta_{\theta_{j}}
\end{aligned}
$$

The random measure $\mathrm{G}$ is a mixture of infinitely many atoms with their weight $w_{j}$ drawn from a stick-breaking distribution. We use iterators $j$ for cluster index and $i$ for data points. The base distribution $H$ defines the locations of atoms $\theta_{j}$, and the concentration parameter $\alpha$ controls the sparseness of G. The base distribution serves as a prior for the data-generating distribution in a mixture model. The concentration parameter $\alpha$ affects the number of components in the mixture model. Larger values of $\alpha$ create smaller initial $w_{j}$ values, giving high probability mass to more clusters. In this notation $\delta_{\theta_{j}}$ represents the impulse function, i.e., atom at location $\theta_{j}$.

\subsection{Chinese Restaurant Process}

In many clustering applications, we are not interested in the actual stick lengths in G. The interesting variables are the labels that create the partitioning of the data. Therefore, $G$ could be integrated out to obtain a prior distribution on partitions of data. This is similar to the finite counterpart of the Dirichletmultinomial compound distribution. This distribution is an exchangeable distribution which ensures that the probability of partition does not depend on permutation of the incoming data. After this integration, the distribution assigns a probability measure for each partition of the data according to (3).

$$
\begin{aligned}
P\left(t_{i}=j\right) & =\frac{n_{j}}{\alpha+N} \\
P\left(t_{i}=K+1\right) & =\frac{\alpha}{\alpha+N}
\end{aligned}
$$

This distribution is metaphorically explained by a Chinese restaurant with an infinite number of tables where $t_{i}$ denotes the table/cluster indicator variables. A new customer is seated at a table with a probability proportional to the number of customers at that table or he is seated at a new table with probability $\alpha$ (Aldous, 1985). In this equation $n_{j}$ denotes the number of points in table $j, K$ is the current number of tables, and $N$ is the total number of points. As data grows, crowded clusters become more crowded, a phenomenon also known as "rich-get-richer". The effect of $\alpha$ is more visible in this representation. For larger values of $\alpha \mathrm{CRP}$ will generate more tables.

\subsection{Uncollapsed Gibbs Sampler}

For Dirichlet Process mixture model, the parameters of mixture components are sampled from the discrete measure $\mathrm{G}$, i.e. $\theta_{j} \sim G$. The posterior distribution of $G$ conditioned on the previously observed $\theta_{j}$ 's can be obtained in a closed form solution (Teh. 2011). This posterior distribution is again a Dirichlet Process with the parameters updated as in (5).

$$
P(G \mid \theta, H, \alpha)=D P\left(\alpha H+\sum n_{j} \delta_{\theta_{j}}\right)
$$

Direct application of Gibbs sampler is not possible for the Dirichlet Process since the measure $\mathrm{G}$ is infinite dimensional. However, this measure could be approximated by a finite number of sticks. This creates an easy-to-sample finite dimensional Dirichlet posterior on sticks. In this sampler, approximated measure $\mathrm{G}$, atom locations $\theta_{j}$, and cluster indicator variables $t_{i}$ would form the state of the sampler. These variables are sampled in turn by a Gibbs sampler. There is an additional difficulty associated with the sampling of the parameters. Empty cluster parameters sampled from $\mathrm{H}$ may not fit the data points well. Thus, the probability of the sampler creating new clusters will get smaller as the dimensionality increases.

$$
\begin{aligned}
t_{i} & \sim P\left(x_{i} \mid \theta_{j}\right) * w_{j} \\
w_{j} & \sim \text { TruncatedStick }(n, \alpha) \\
\theta_{j} & \sim P\left(\theta_{j} \mid X\right)
\end{aligned}
$$

Truncated stick distribution is the finite length form of the stick-breaking distribution in $(2)$. The notation $P\left(\theta_{j} \mid X\right)$ represents the posterior distribution on cluster parameters of cluster $j$ where $X$ denotes the set of points assigned to cluster $j$.

\subsection{Collapsed Gibbs Sampler}

In the collapsed version of the sampler, hidden variables in the model need to be integrated out. For a conjugate family of prior and data distributions, integration of intermediate parameters produces a closed form solution of the predictive distribution. Also, integration of the Dirichlet Process measure G creates the CRP distribution. After these integrations, the Gibbs sampler for the CRP mixture will have only the cluster indicator variables in the state of the sampler. The probability of each cluster can be defined based on the CRP prior and the posterior predictive data likelihood as in (9).

$$
\begin{aligned}
P\left(t_{i}=j \mid X, T\right) & =n_{i} * P\left(x_{i} \mid X, t_{i}=j\right) \\
P\left(t_{i}=K+1 \mid X, T\right) & =\alpha * P\left(x_{i}\right)
\end{aligned}
$$

The notation $P\left(x_{i} \mid X, t_{i}=j\right)=\int P\left(x_{i} \mid \theta_{j}\right) P\left(\theta_{j} \mid X\right) d \theta_{j}$ indicates the posterior predictive likelihood of data point $x_{i}$ for existing clusters. $P\left(x_{i}\right)$ is the posterior predictive likelihood for an empty cluster. This likelihood does not depend on other data points since no other point is previously assigned to this cluster. These marginal distributions are obtained in the form of student- $t$ distributions for a Normal $\times$ Inverse Wishart prior and Normal data model (NNIW). For the collapsed Gibbs sampler, it is a common practice to show the exclusion of the data point whose cluster indicator variable is being sampled by a superscript notation as $T^{-i}$ but we avoid this notation to keep our notation simple. From hereon $T$ in the conditional part indicates $T^{-i}$.

\section{Combining CRP with Stick-Breaking}

We start by defining the representation that combines Chinese restaurant process with a stick-breaking prior. Details of the sampling process are described in the next section. 
Definition 2 (DP Decomposition). We define a discrete measure $G$ according to the following generative model

$$
\begin{array}{r}
\left(w_{1}, \ldots, w_{K}, w_{\alpha}\right) \sim \operatorname{Dir}\left(n_{1}, \ldots, n_{K}, \alpha\right) \\
G_{j} \sim \operatorname{DP}\left(n_{j} \delta_{\theta_{j}}\right) \\
G_{\alpha} \sim \operatorname{DP}(\alpha H) \\
G=\sum_{j=1}^{K} w_{j} G_{j}+w_{\alpha} G_{\alpha}
\end{array}
$$

\section{Theorem 1.}

$$
G \sim D P\left(\alpha H+\sum_{j=1}^{K} n_{j} \delta_{\theta_{j}}\right)
$$

Proof. Similar versions of this proof were introduced in the literature previously. The most recent one gives a general proof that applies to this special case as well (Williamson et al. 2013). The main part of the proof follows from the fact that the superposition of Gamma processes is another Gamma process. Normalization followed by the superposition of Gamma processes creates a Dirichlet process. Also, one can relate this representation to the generalization lemma of Sethuraman's stick-breaking construction (Sethuraman, 1991).

The main difference of our approach from earlier DP representations discussed in the literature lies in the approximation of the small residual part $G_{\alpha}$ by its expectation. This way the representation creates completely independent distributions leading to a fully parallelizable inference. Other decomposition approaches for parallelism uses identical Dirichlet Process distributions to separate data into multiple cores. As (Gal and Ghahramani, 2013) states these yield an unbalanced task distribution.

The summation of $\sum w_{j} G_{j}$ constitutes the observed part of the decomposition whereas $G_{\alpha}$ is the unobserved part. Unit DP's of the observed part $G_{j}=\delta_{\theta_{j}} \sim D P\left(n_{j} \delta_{\theta_{j}}\right)$ reduces this part into a finite discrete/multinomial measure. Since the atom locations are fixed during a single scan, likelihood calculations for all data points can be carried out independent from each other paving the way for parallelization. As a weighted summation of discrete measures, this decomposition defines a mixture of DPs, which itself is another DP. We can define a latent variable $z_{i}$ to choose a DP associated with the data point $x_{i}$. Calculating the probability of $z_{i}$ for the observed part is trivial since all DPs in this part are unit DPs. For the unobserved part, we integrate out cluster parameters and calculate the marginal likelihood of $z_{i}$ belonging to $G_{\alpha}$. This is calculated by approximating $G_{\alpha}$ by its expectation H. All data points associated with $G_{\alpha}$ are sampled again using a CRP and original DP is reconstructed for the next iteration. As the sampler converges to the stationary distribution number of data points handled by the unobserved part will gradually decrease.

\section{Partially Collapsed Sampler}

The inference technique we use for the proposed representation of DP is from the family of Gibbs samplers, which involves

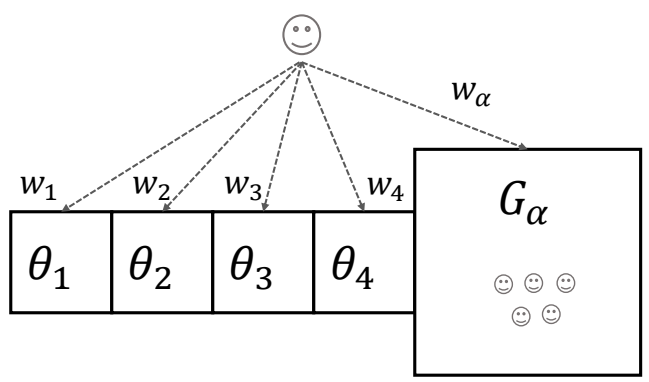

(a) Sampling Dirichlet Process indicators

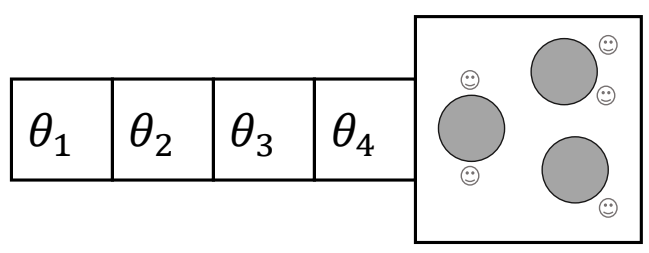

(b) Sampling new clusters

Figure 1: Partially collapsed Gibbs sampler

a combination of collapsed and uncollapsed Gibbs samplers hence the name partially collapsed sampler. The uncollapsed part of the sampler deals with the summation in (14) involving the unit DPs. For this part, the state of the sampler contains DP indicator variables, stick weights, and cluster parameters. The collapsed part deals with the unobserved part of DP involving $G_{\alpha}$. In this part, the state of the sampler only contains the cluster indicators which can be sampled as described in Section 3.4 For the uncollapsed Gibbs sampler, cluster parameters are sampled from their corresponding posterior distributions given the cluster allocations for all data points and prior distribution $\mathrm{H}$. This is similar to the uncollapsed sampler described in Section 3.3 Stick weights are the parameters of the multinomial distribution associated with measure $\sum_{j=1}^{K} w_{j} \delta_{\theta_{j}}$. These weights are sampled from the Dirichlet distribution in (11). Sampling of the cluster parameters and stick weights does not introduce a significant computational burden. The proposed representation plays a critical role in the sampling of DP indicators $z_{i}$. Since unit DPs are producing the same parameter each time, the likelihood of a data point belonging to a specific unit DP depends only on the corresponding cluster parameter, which is fixed until the next scan of the Gibbs sampler. The likelihood of the data point for an empty CRP only depends on the expected $G_{\alpha}$, which is $H$ in the proposed representation. Thus, the sampling of $z_{i}$ 's can be carried out independent of one another. This sampling operation is illustrated in Figure 1 .

The most computational part of the proposed Gibbs sampler involves the sampling of $z_{i}$ 's but thanks to the proposed decomposition of DP this sampling can be performed in parallel. Conditioned on the cluster parameters and stick weights cluster conditional likelihoods for all data points can be computed independently. This offers an important opportunity to parallelize 
repeat

- Sample :

- $\boldsymbol{w} \sim \operatorname{Dir}\left(n_{1}, n_{2}, \ldots, n_{K}, \alpha\right)$

- $\theta_{j} \sim P\left(\theta_{j} \mid \boldsymbol{X}\right)$

- DP indicators (sample in parallel)

$z_{i} \sim\left\{\begin{array}{l}w_{j} P\left(x_{i} \mid \theta_{j}\right) \text { if } z_{i}=j \\ w_{\alpha} P\left(x_{i}\right) \text { if } z_{i}=K+1\end{array}\right.$

- For $\forall z_{i}=(K+1)$ sample cluster indicators associated with $G_{\alpha}$

- Sample cluster indicators $t_{i}$ using CRP based collapsed sampler in equation 9

- Reconstruct the Dirichlet process for the next iteration by combining observed and unobserved parts.

until convergence or for a designated number of iterations; Algorithm 1: Parallel Partially Collapsed Gibbs Sampler

the most computational part of the inference process with very high granularity. The central part of the probability mass of the posterior stays in the uncollapsed part of the sampler. The remaining part is modeled by a CRP associated with $G_{\alpha}$. Collapsing this part alleviates the problem of creating new clusters by the uncollapsed sampler as discussed in Section 3.3 .

With the proposed sampler, the uncollapsed part is executed for all data points during each scan of the sampler. However, the collapsed part is only called when there are data points identified as potentially belonging to new clusters during the uncollapsed part. During the first few iterations of the sampler more of the data points could be identified this way for which the collapsed part has to be run. Running the collapsed sampler during a scan may increase the computational time required to complete that scan. However, as the sampler gradually mixes the collapsed part, it will process less and less number of data points reducing the computational time required to complete a scan. Also, this collapsed sampling allows sampler to be adaptive for small clusters and creates more accurate clustering.

\section{Results}

We compared the proposed parallel partially collapsed Gibbs inference (PPCG) technique against the standard collapsed Gibbs sampler (CG) as well as two parallel uncollapsed Gibbs samplers: inference with sub-cluster splits (SUBC) (Chang and Fisher III, 2013) and parallel slice sampler (PSS) (Ge et al. 2015). We used a Normal data model and a Normal $\times$ Inverse Wishart base distribution. This model involves five hyperparameters, namely, the scale matrix $\Psi$ and the degrees of freedom $m$ of the Inverse Wishart, the mean $\mu_{0}$ and dispersion parameter $\kappa_{0}$ of the Normal prior, and the concentration parameter $\gamma$ of the DP prior. For these hyperparameters, we used the same values given in (Chang and Fisher III, 2013). More specifically, $\kappa_{0}=1$, $\Psi=I * m, m=d+3, \gamma=1$, and $\mu_{0}$ is set to the mean of the data.
Previous works use data likelihood for performance evaluation. Although likelihood could be considered an effective performance measure for density estimation problems, it is suboptimal for clustering problems. We believe that mean $F_{1}$ score would be a more effective indicator of clustering accuracy. For each technique, the sampler runs for 500 scans 300 of which are ignored as burn-in. Samples are drawn at every 20 iterations to obtain a total of 10 samples of cluster labels. These labels are aligned using the Hungarian method (Cron and West, 2011) and result is used for final evaluation.

We used two image datasets (MNIST and CIFAR10), a text corpus (20News), and a hyper-spectral image dataset (Indian Pines). MNIST data contains 70K digit images. For CIFAR10 we used only the training set containing 50k images. The $20 \mathrm{News}$ data set contains around $16 \mathrm{~K}$ data points each characterizing a news article 1 Indian Pines dataset contains around $10 \mathrm{~K}$ labeled pixels each represented by 200 channels. We preprocessed all datasets by PCA whitening and use the first fifty principal components to reduce dimensionality in line with previous studies. All experiments are run on a 8-core 64-bit Windows machine.

Each experiment is run ten times and average $F_{1}$ scores along with standard deviations are given in table 1 . We also noted number of clusters generated for each method in table 2.

Before we discuss the performances of PPCG and SUBC algorithms it is essential to recall the differences and similarities between these two techniques. The proposed PPCG algorithm uses a combination of uncollapsed and collapsed Gibbs sampler with the main sampling process being centered on the uncollapsed part. The collapsed part is only run when the uncollapsed sampler identifies data points as potentially not belonging to any of the existing clusters. SUBC algorithm also uses an uncollapsed Gibbs sampler as the main sampler but to achieve a better mixing the main sampler is backed by split-merge proposals, which are evaluated every hundred iterations on the average. Thus, the main difference in PPCG over SUBC is that splitmerge steps in SUBC are replaced by the collapsed sampler.

On the CIFAR10 dataset, both PPCG and SUBC generates similar number of clusters and take more or less same amount of time to complete 500 iterations. PPCG achieves an $F_{1}$ score of 0.33 versus 0.30 by SUBC. On the MNIST data set SUBC generates almost three times more clusters than PPCG and takes three times more to complete 500 iterations. For this dataset, PPCG achieves an $F_{1}$ score of 0.67 versus 0.53 by SUBC. On the 20News data set PPCG generates more clusters than SUBC but finishes 500 iterations faster than SUBC. For this dataset, PPCG achieves an $F_{1}$ score of 0.56 versus 0.44 by SUBC.

When PPCG is compared against PSS we observe significantly higher F1 scores for three of the four data sets favoring PPCG in terms of clustering accuracy. Although PSS is the fastest algorithm among the three parallel samplers, significant deterioration in clustering accuracy compared to PPCG and SUBC is considered a major concern.Also, experimental results suggest that PSS is highly sensitive to the initial clustering con-

${ }^{1}$ Matlab version of 20News dataset is taken from Sam Roweis' web page http://www.cs.nyu.edu/ ${ }^{\sim}$ roweis/data.html 
Table 1: Clustering comparison on real world datasets

\begin{tabular}{lllllllll}
\hline \multicolumn{5}{c}{$\bar{F}_{1}$} & \multicolumn{4}{c}{ Elapsed Time $(\mathrm{m})$} \\
\hline Dataset & PPCG & SUBC & PSS & CG & PPCG & SUBC & PSS & CG \\
\hline Cifar10 & $0.33(0.01)$ & $0.30(0.01)$ & $0.24(0.01)$ & $0.29(0.01)$ & 8.30 & 22.33 & 9.20 & 56.79 \\
\hline Mnist & $0.67(0.03)$ & $0.53(0.01)$ & $0.53(0.01)$ & $0.70(0.05)$ & 5.95 & 19.19 & 11.49 & 54.48 \\
\hline 20News & $0.56(0.01)$ & $0.44(0.04)$ & $0.41(0.02)$ & $0.57(0.01)$ & 3.46 & 14.02 & 2.16 & 15.01 \\
\hline Pines & $0.31(0.04)$ & $0.29(0.02)$ & $0.29(0.02)$ & $0.35(0.01)$ & 1.77 & 22.04 & 1.53 & 8.94 \\
\hline
\end{tabular}

Table 2: Number of clusters in the experiments

\begin{tabular}{lllll}
\hline Dataset & PPCG & SUBC & PSS & CG \\
\hline Cifar10 & 18.20 & 17.10 & 48.40 & 21.10 \\
\hline Mnist & 14.10 & 37.10 & 42.10 & 14.20 \\
\hline 20News & 26.40 & 17.40 & 48.70 & 13.50 \\
\hline Pines & 17.80 & 13.10 & 33.70 & 11.40 \\
\hline
\end{tabular}

figuration. We found out that the final number of clusters is not much different than the initial number of clusters especially in high dimensions, which indicates the sampler cannot make significant moves between successive Gibbs scans and seems to be more vulnerable to local optima problem than the other two samplers.

When we summarize results across all four data sets we observe a consistent trend favoring the proposed PPCG algorithm in terms of $F_{1}$ scores as compared to other parallel algorithms. Interestingly this is achieved despite other algorithms converging to higher steady-state likelihood values in all four data sets.

These results support our conviction that likelihood is not a good measure for evaluating clustering performance. Poorly recovered small clusters may not affect likelihood as much as larger ones but may still have significant impact on the clustering performance especially when performance is measured by mean $F_{1}$ score.

Run time results also favor PPCG as compare to SUBC and collapsed sampler. With additional number of cores ( 8 is used) parallel algorithms would have more speed up as compare to the collapsed sampler. However, we believe that not all of this difference can be attributed to the difference in sampling algorithms as there are other factors affecting run-time in the current settings. Although all algorithms are implemented in $\mathrm{C}++, \mathrm{PPCG}$ and SUBC uses different numerical optimization techniques for matrix inversion. Inversion is done once per iteration for parallel sampler. However, collapsed sampler recomputes the inverse of the covariance matrix every time a point is removed or added to a cluster. Also, the number of clusters generated during the runtime can be significantly different between algorithms, which also affects run-time. One interesting case as far as run-time comparison is concerned is the 20NEWS data set where PPCG generates more clusters yet takes less amount of time than SUBC to complete the same number of iterations. When we further scrutinize the distributions of cluster sizes for both algorithms we find that most of the clusters generated by PPCG have less than 10 points whereas the smallest cluster generated by SUBC contains 16 points. If we ignore smaller clusters we can explain the difference in computation time by the larger number of clusters generated by SUBC similar to the MNIST case. For a more conclusive comparison of run-time between PPCG and SUBC, an experiment with a big dataset and on a computer with a large number of cores would be desirable, which we assign as a future task.

The computational times of PSS and PPCG are comparable. In general we expect PSS to have a slightly faster sampling time as the sampler in this approach calculates the likelihood of a table only when the prior weight is above the threshold defined by the auxiliary slice variable. However, for some data sets this slight computational gain may be lost due to the higher number of clusters generated.

\section{Conclusion}

We proposed a decomposition for DP that combines stickbreaking construction and CRP representation into a single representation. We used a partially collapsed Gibbs sampler for inference in DP mixture models that relies on this representation. Stick-breaking part of the proposed decomposition enables parallelization at the data point level whereas collapsed part handles new clusters. We presented results with four real-world datasets that show up to an order of magnitude improvement in computational time compared to collapsed-only sampler and significantly higher $F_{1}$ scores compared to existing parallel samplers.

We believe that this method can be extended to dependent DP models such as HDP or nCRP (Teh and Jordan, 2010, Blei et al. 2004). Additional experiments on big datasets using machines with large number of cores and threads are needed to more effectively evaluate the proposed sampler against state-of-the-art parallel and distributed samplers.

The source codes for CG, PPCG, and PSS are available through GitHub ${ }^{2}$. The source code for SUBC can be obtained from Chang's website 3 .

\footnotetext{
https://github.com/halidziya

http://people.csail.mit.edu/jchang7/code
} 


\section{Acknowledgments}

This research was sponsored by the National Science Foundation (NSF) under Grant Number IIS-1252648 (CAREER). The content is solely the responsibility of the authors and does not represent the official view of NSF.

We would like to thank Dr. Vinayak Rao for insightful discussions.

\section{References}

Aldous, D. J., 1985. Exchangeability and related topics. In: École d'Été St Flour 1983. Springer-Verlag, pp. 1-198, lecture Notes in Math. 1117.

Blei, D. M., Griffiths, T. L., Jordan, M. I., Tenenbaum, J. B., 2004. Hierarchical topic models and the nested chinese restaurant process. In: Advances in Neural Information Processing Systems. MIT Press, p. 2003.

Chang, J., Fisher III, J. W., 2013. Parallel sampling of dp mixture models using sub-cluster splits. In: Advances in Neural Information Processing Systems. pp. 620-628.

Cron, A. J., West, M., 2011. Efficient classification-based relabeling in mixture models. The American Statistician 65, 16-20, pMC3110018. URL http://ftp.stat.duke.edu/WorkingPapers/10-20.html

Dahl, D. B., 2003. An improved merge-split sampler for conjugate dirichlet process mixture models. Technical R eport 1, 086.

Ferguson, T. S., 1973. A bayesian analysis of some nonparametric problems. The annals of statistics, 209-230.

Gal, Y., Ghahramani, Z., 2013. Pitfalls in the use of parallel inference for the dirichlet process. In: In Workshop in Big Learning, NIPS.

Ge, H., Chen, Y., Wan, M., Ghahramani, Z., 2015. Distributed inference for dirichlet process mixture models. In: Proceedings of the 32nd International Conference on Machine Learning (ICML-15). pp. 2276-2284.

Görür, D., Rasmussen, C. E., 2010. Dirichlet process gaussian mixture models: Choice of the base distribution. Journal of Computer Science and Technology 25 (4), 653-664.

Ishwaran, H., James, L. F., 2001. Gibbs sampling methods for stick-breaking priors. Journal of the American Statistical Association 96 (453), pp. 161-173. URL http://www.jstor.org/stable/2670356

Ishwaran, H., James, L. F., 2011. Gibbs sampling methods for stick-breaking priors. Journal of the American Statistical Association.

Jain, S., Neal, R. M., 2004. A split-merge markov chain monte carlo procedure for the dirichlet process mixture model. Journal of Computational and Graphical Statistics 13 (1).

Jain, S., Neal, R. M., et al., 2007. Splitting and merging components of a nonconjugate dirichlet process mixture model. Bayesian Analysis 2 (3), 445472.

Lovell, D., Adams, R. P., Mansingka, V., 2012. Parallel markov chain monte carlo for dirichlet process mixtures. In: Workshop on Big Learning, NIPS.

Neal, R. M., 2000. Markov chain sampling methods for dirichlet process mixture models. Journal of computational and graphical statistics 9 (2), 249-265.

Papaspiliopoulos, O., Roberts, G. O., 2008. Retrospective markov chain monte carlo methods for dirichlet process hierarchical models. Biometrika 95 (1), 169-186.

Sethuraman, J., 1991. A constructive definition of dirichlet priors. Tech. rep., DTIC Document.

Teh, Y. W., 2011. Dirichlet process. In: Encyclopedia of machine learning. Springer, pp. 280-287.

Teh, Y. W., Jordan, M. I., 2010. Hierarchical bayesian nonparametric models with applications. Bayesian nonparametrics 1 .

Williamson, S., Dubey, A., Xing, E., 2013. Parallel $\{M\}$ arkov chain $\{M\}$ onte $\{C\}$ arlo for nonparametric mixture models. In: Proceedings of the 30th International Conference on Machine Learning. pp. 98-106. 